\title{
Portal Vein Thrombosis, CTCAE
}

National Cancer Institute

\section{Source}

National Cancer Institute. Portal Vein Thrombosis, CT CAE. NCI Thesaurus. Code

C143770.

A disorder characterized by the formation of a thrombus (blood clot) in the portal vein. 